Nouvelle Revue Synergies Canada

\title{
Les lettres-objets de Simone de Beauvoir : entretien avec Sylvie Le Bon de Beauvoir
}

\section{Karin Schwerdtner}

Numéro 13, 2020

Objets de l'écrivain : images, usages, représentations depuis le XIXe siècle à nos jours

URI : https://id.erudit.org/iderudit/1078435ar

DOI : https://doi.org/10.21083/nrsc.vi13.5364

Aller au sommaire du numéro

Éditeur(s)

University of Guelph, School of Languages and Literatures

ISSN

2292-2261 (numérique)

Découvrir la revue

Citer ce document

Schwerdtner, K. (2020). Les lettres-objets de Simone de Beauvoir : entretien avec Sylvie Le Bon de Beauvoir. Nouvelle Revue Synergies Canada, (13), 1-9. https://doi.org/10.21083/nrsc.vi13.5364 d'utilisation que vous pouvez consulter en ligne. 


\title{
Les lettres-objets de Simone de Beauvoir: entretien avec Sylvie Le Bon de Beauvoir ${ }^{1}$
}

\author{
Karin Schwerdtner \\ Université Western Ontario \\ Canada
}

Depuis plusieurs décennies, historiens de l'intime et critiques littéraires constatent un intérêt accru en France pour la publication et l'étude des papiers privés. Parmi d'autres formes d'écriture intime, les lettres, en particulier, bénéficient d'une attention toujours plus importante, à mesure que se multiplient en même temps les publications de correspondances et les articles de presse et ouvrages scientifiques qui s'y intéressent. $^{2}$ Entre autres correspondances que, pour leur part, les éditions Gallimard ont publiées se trouvent - en plusieurs volumes établis par Sylvie Le Bon de Beauvoir, fille adoptive de Simone de Beauvoir et héritière de son œuvre et de ses papiers et manuscrits - , les lettres de la grande écrivaine, qui fut aussi une grande épistolière: Lettres à Sartre (1990), Lettres à Nelson Algren (1997), et Correspondance croisée avec Jacques-Laurent Bost (2004). À l'éditrice scientifique de ces correspondances, nous devons également l'Album, conçu, en partie à partir de lettres inédites, pour accompagner la publication des Mémoires (2018) de Beauvoir dans la Pléiade. Pour ce qui est des lettres inédites que l'auteure du Deuxième sexe (1947) a reçues d'amis, d'écrivains et de lecteurs de « toutes les nationalités, toutes les classes sociales " (comme le rappelle ici Sylvie Le Bon de Beauvoir), la plupart d'entre elles se trouvent, depuis 1995, au Département des Archives et Manuscrits de la Bibliothèque nationale de France, dans le Fonds Simone de Beauvoir: seules les lettres de célébrités et d'intimes sont archivées chez l'héritière. C'est en effet grâce au don (ou à la dation, selon le cas), fait par Sylvie Le Bon de Beauvoir, qu'il est possible pour nous de « lire les objets " (Pomel) que sont les missives envoyées à l'une des écrivains marquants $\mathrm{du} X \mathrm{X}^{\mathrm{e}}$ siècle. $\mathrm{Si}$, par ailleurs, nous pouvons consulter à la BNF la correspondance Sartre-Beauvoir, c'est qu'elle a fait, quant à elle, l'objet d'une dation, ce que notre interviewée précise dans l'entretien qui suit.

Nos questions pour l'auteure de l'Album, qui nous a reçu chez elle, à Paris, le 8 novembre 2018, portent sur son travail de tri ${ }^{3}$ et d'édition, sur les circonstances qui ont amené la publication des Lettres à Sartre entre autres, sur le cadeau que Simone de Beauvoir nous a fait en conservant ses "lettres reçues ${ }^{4}$ ", et sur le don grâce auquel, aujourd'hui, il est possible de les lire. Au terme de cet entretien, nous avons bien compris combien, pour sa fille adoptive, "Simone de Beauvoir était quelqu'un d'une rare générosité ${ }^{5}$ ". Non seulement elle donnait énormément d'elle-même, de son temps, en prêtant attention et en écrivant à autrui, mais aussi, à mesure que se développait son goût de tout conserver, Simone de Beauvoir nous a laissé une véritable somme de papiers privés, qui sont autant de traces de sa pensée à différents moments de sa vie, et dont la lecture aujourd'hui permet de découvrir ou de retrouver la " grande » Simone de Beauvoir dans toute son humanité.

KS : Simone de Beauvoir vous a confié la gestion de son œuvre et, en particulier, de ses papiers privés, parmi lesquels vous avez publié, en 1990, Lettres à Sartre, en 1997, Lettres à Nelson Algren et, en 2004, Correspondance croisée avec Jacques-Laurent Bost. Pourriez-vous nous décrire votre travail, dans ce cadre?

SLB : Lorsque Simone de Beauvoir est décédée le 14 avril 1986, ce fut pour moi un écroulement. La mission qu'en m'adoptant elle m'avait confiée - m'occuper de son œuvre quand elle ne serait plus là - m'a sauvée. Son œuvre déjà publiée mais aussi ses nombreux inédits; parmi ces derniers, elle m'avait signalé des correspondances et des journaux intimes, en me suggérant des directives. Très vite, dès novembre, je me suis mise à transcrire ses Lettres à Sartre. II est notoire que sa graphie est très difficile à déchiffrer, je le savais et y étais accoutumée mais malgré cela je butais, parfois obstinément, sur des mots illisibles. J'ai trouvé logique de commencer par ses Lettres à Sartre, puisqu'elle-même avait publié peu de temps auparavant, en 1983, les Lettres au Castor, c'est-à-dire les lettres que Sartre lui avait adressées. Ensuite les circonstances ont été déterminantes : les lettres de Simone de Beauvoir à Nelson Algren se trouvaient dans une Université de l'Illinois, et diverses personnes qui avaient commencé à les transcrire, à cause de la difficulté de son écriture manuscrite, n'en venaient pas à bout. II y avait des aberrations dans ce que j'en voyais. J'ai senti avec inquiétude à quel point ces lettres étaient exposées au pillage et qu'il fallait couper court à toute infidélité possible en entreprenant moi-même une version autorisée. Comme Simone de Beauvoir écrivait en anglais à Algren, s'ajoutaient aux difficultés de la transcription proprement dite celles de la traduction en français. II fallait respecter non seulement la littéralité, qui est évidemment primordiale, mais aussi l'esprit du texte. II fallait faire vivre cette correspondance, ressusciter le ton, le rythme propres à l'épistolière, ses idiotismes, ses expressions familières, ses jeux, voire ses tics de langage, par exemple son goût caractéristique pour l'adjectif «plaisant». Or j'avais, j'ai encore dans l'oreille sa voix, ses 
Schwerdtner, Karin. «Les lettres-objets de Simone de Beauvoir : entretien avec Sylvie Le Bon de Beauvoir. » Nouvelle Revue Synergies Canada, №13 (2020)

intonations, cette expression parlée qui m'enchantait, riche d'inventions drôles et de mots de passe, avec ses modulations inattendues de l'argot d'étudiant aux allusions littéraires raffinées (ainsi « battu de l'oiseau » est emprunté à Saint-Simon, etc.)

Pour la Correspondance croisée, il se trouve que Jacques-Laurent Bost était un très grand ami à moi. Un jour il est arrivé en me tendant un petit paquet: c'était les lettres que Simone de Beauvoir lui avait envoyées. II m'a dit : "C'est toi qui dois avoir ça ". J'ai été émerveillée. Comme elle-même avait conservé celles de Bost, j'ai pu réaliser une correspondance croisée.

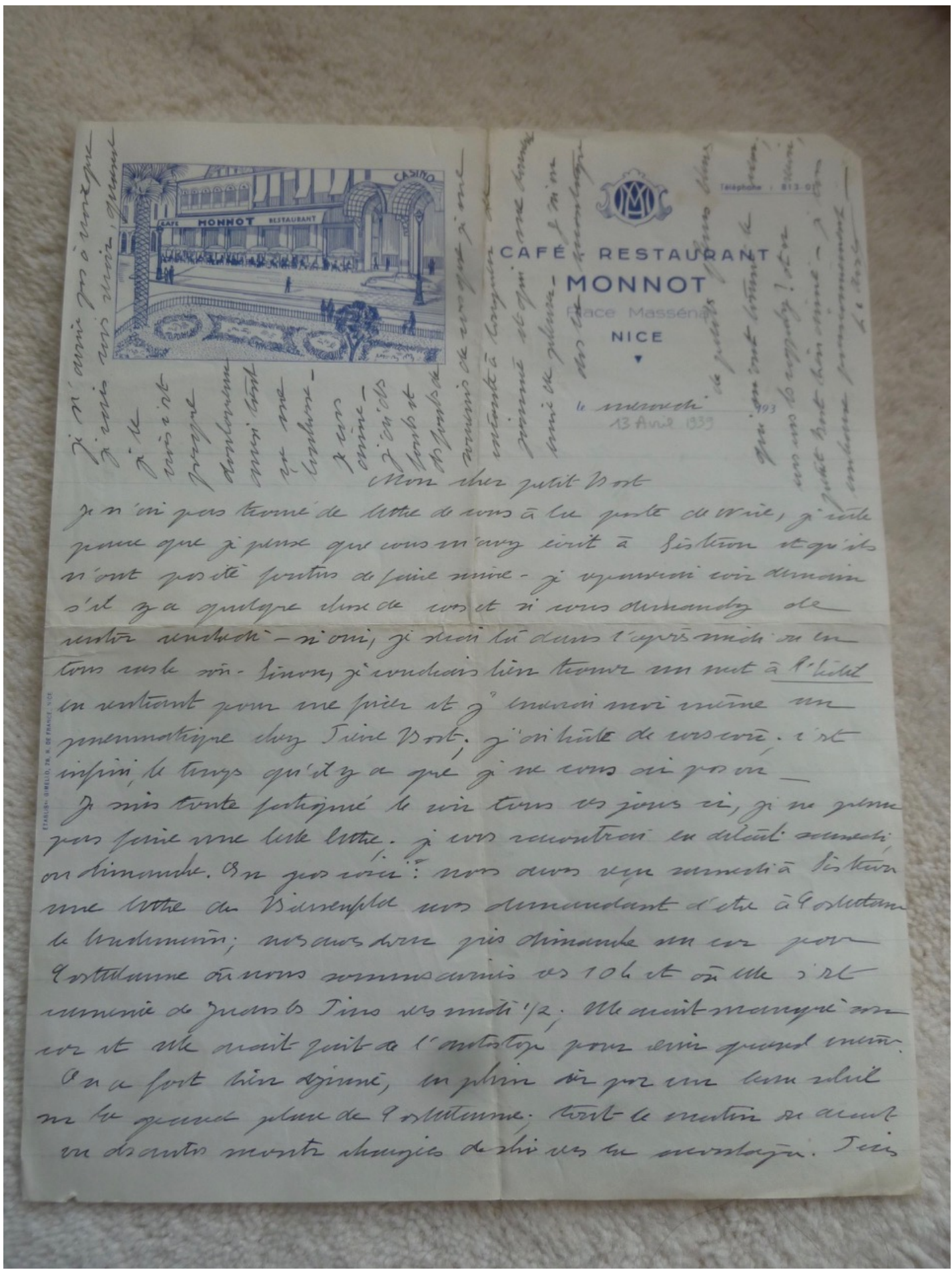

Lettre à Bost 1939. Collection privée de Sylvie Le Bon de Beauvoir. Photo reproduite avec sa permission. 
Simone de Beauvoir a été une très grande épistolière. Outre ce qui fait partie de ses archives, il existe quantité d'inédits de par le monde. Les hasards, les rencontres, les circonstances continuent à jouer un grand rôle, des lettres ressurgissent ici et là, les destinataires ou leurs héritiers m'en envoient spontanément copie, certaines apparaissent dans des ventes publiques, j'en découvre dans des bibliothèques universitaires ou des musées. Récemment, j'ai eu accès aux lettres de jeunesse échangées avec Maurice Merleau-Ponty. L'ensemble le plus important est peut-être celui des lettres à sa mère, mis en vente chez Christie's en 2014. En me battant, j'ai pu en avoir communication. Combien j'ai rendu grâce à Simone de Beauvoir de m'avoir adoptée - au début j'étais réticente - car même avec le poids de la loi, même en étant son héritière incontestée, il faut se battre pour faire respecter ses droits!

Dès sa jeunesse, dès l'enfance presque, la correspondance accompagne le rapport de Simone de Beauvoir aux autres. Bien entendu, au cours de sa vie, le sens en a évolué. Au début, c'est une correspondance purement privée, intime, avec Sartre, avec Olga Kosackiewicz, avec les hommes qu'elle a aimés. Par la suite, la dimension publique prend le dessus, liée à la littérature, à son œuvre, à sa dimension d'écrivain et de penseur, aux débats et aux événements politiques. À mesure qu'elle avançait dans sa carrière, elle a disposé de moins de loisir pour les correspondances gratuites, de plaisir, même ses amitiés étaient engagées dans le cours du monde. Des lecteurs, toutes sortes de lecteurs lui écrivaient. Elle répondait à tous, elle y tenait, ce qui n'a rien de surprenant étant donné le goût, l'intérêt vivant et le respect qu'elle avait pour les autres. Elle y parvenait grâce à sa remarquable discipline de travail : les matinées consacrées à l'écriture littéraire, d'autres moments de la journée, limités, réservés au courrier. Rédigé par elle à la main, je le rappelle, car elle n'a jamais usé d'une machine à écrire, ni employé, comme Sartre, de secrétaire.

\section{KS : Soucieuse de toujours répondre, avait-elle aussi le sentiment d'une obligation, selon vous?}

SLB : Oui, mais pas au sens où ce serait une corvée. Elle se faisait un devoir de donner de son temps et de son énergie pour répondre personnellement à tous ses correspondants, mais c'était aussi un plaisir. Elle aimait ces échanges avec toutes sortes de gens, lectrices et lecteurs de toute nationalité, de toute classe sociale, de tout âge. Elle était heureuse de recevoir un tel courrier, qui lui semblait une facette importante de sa vie d'écrivain. II en était autrement pour Sartre, c'est une de leurs nombreuses différences. Parler aux autres « de bouche à oreille " est, on le sait, une des motivations profondes de l'œuvre de Simone de Beauvoir. Bien entendu elle avait des ennemis et recevait des missives hostiles, mais la plupart étaient la preuve vivante, indubitable, qu'elle parvenait à toucher son public, que beaucoup l'aimaient et l'admiraient, que son œuvre servait - et c'est cela qu'elle avait voulu. Cela comptait beaucoup plus pour elle que l'avis des critiques professionnels. On sent la force de ce lien original, unique, avec son public, dans cette correspondance avec ses lecteurs, qu'en certains cas elle accompagnait pendant de longues années et dont certains lui livraient avec confiance ce qu'ils n'ont parfois livré à personne d'autre. On retrouve ici sa générosité, son talent pour la réciprocité : elle prend la peine d'expliquer sa pensée, ses choix, et elle s'implique chaleureusement dans ceux de ses interlocuteurs. Ce n'est pas un hasard, mais une attitude fondamentale chez elle.

\section{KS : Pour votre part, échangiez-vous souvent par lettre avec Simone de Beauvoir?}

SLB : C'est ainsi que nos relations ont commencé, par correspondance. Admiratrice de ses romans, je lui ai écrit - bien maladroitement - quand je suis venue faire mes études à Paris. Quand nous avons été liées, lors de ses absences de Paris, surtout lors de ses longs voyages semi officiels, en Égypte, au Japon, nous nous écrivions beaucoup et en plus, elle me demandait de tenir un journal, qui tiendrait lieu de nos conversations quasi quotidiennes. Cette correspondance me semble encore mon bien privé, je la garde jalousement, je n'aimerais pas l'exposer au grand jour - ce qui est peut-être blâmable. Je l'ai transcrite, numérisée et imprimée, parce que je crains qu'on ne puisse pas la lire ou avec des erreurs. Car il faut avouer que recevoir une lettre d'elle était une épreuve, à la fois un bonheur et une torture. Avide de la lire, on ouvrait fébrilement l'enveloppe et là, désespoir! On tombait sur des hiéroglyphes! On désespérait un peu. Sartre, Bost s'en plaignent aussi. Sartre disait qu'il fallait avoir les yeux de l'amour pour la lire. Mais on finissait par tout déchiffrer ...

KS : Vous arrive-t-il, aujourd'hui encore, de dédier du temps à la correspondance épistolaire? Et combien d'heures consacrez-vous par jour en moyenne au travail de transcription des lettres?

SLB : Hélas, la messagerie électronique a tué le courrier postal, on ne peut que le constater. Autrefois j'adorais écrire de longues lettres, aujourd'hui j'en envoie très peu. Certes, je continue la correspondance, mais j'ai cédé aux séductions de l'ordinateur et du mailing : c'est quand même extraordinaire de recevoir 
en France, en quelques minutes, une réponse d'Australie ou du Canada! Mais ce n'est pas du tout la même chose que le tête-à-tête avec du papier et un stylo et l'attente de la réponse.

Les transcriptions, je m'y consacre de façon irrégulière. II y a des périodes de frénésie, où je travaille dix à douze heures de suite, impossible de m'en arracher. Et si un mot résiste, il m'arrive de me relever la nuit pour vérifier une hypothèse. Ces mots récalcitrants, je les photographie, je les magnifie, et à la longue je les déchiffre. S'acharner est contre-productif, il faut les laisser de côté et y revenir. Dans le cas des lettres à Algren, parce qu'elles sont écrites en anglais, il y avait une difficulté supplémentaire : même si je les lisais, je ne reconnaissais pas certains mots. Simone de Beauvoir rédigeait en anglais de chic, au courant de la plume, son vocabulaire était riche (dès l'enfance elle a lu en cette langue). Moi, je devais recourir à des dictionnaires. Trop souvent, de plus, elle ne datait pas et une main inconnue, à l'Université de l'lllinois, avait introduit des dates erronées qui m'ont retardée : je me suis trouvée face à des casse-tête. II a fallu remettre de l'ordre, de la logique, par recoupement avec d'autres documents. C'est pourquoi cette édition m'a pris beaucoup plus de temps que les autres. D'autant plus que j'avais établi une correspondance croisée, traduit et incorporé les réponses d'Algren, qui contiennent des expressions de slang américain et des plaisanteries souvent obscures. Comme finalement nous n'avons pas obtenu les droits d'édition, j'ai dû les retirer et repenser le tout, d'où une nouvelle prolongation (sept ans en tout).

KS : Selon Le Monde, les Lettres à Nelson Algren se seraient «écoulées à 25000 exemplaires en grand format, avant de connaître un nouveau succès en édition de poche " (Bouchy). Quelles raisons justifient le succès de ces lettres aujourd'hui? Selon vous, le public est-il nostalgique d'une époque où la correspondance était " un instant privilégié pour soi comme pour ses relations avec autrui » ${ }^{6}$ ?

SLB : Oui, et je crois que Simone de Beauvoir partageait ce sentiment. Ce fut, je le répète, une grande épistolière. Ce qu'elle trouvait précieux dans les lettres c'est qu'on peut, davantage que dans la littérature, que dans un roman en particulier, qui doit créer un univers nécessaire, s'y permettre d'y refléter la contingence du vécu, ce qu'elle appelait «la poussière du quotidien ». Dans ses Mémoires, elle nous confie qu'elle a entretenu longtemps le fantasme que tous les instants de son existence seraient sauvés quelque part, sur un magnétophone géant. Les lettres précisément enregistrent cette " poussière " qui va disparaître à jamais. Comme lectrice, elle avait le goût des correspondances du passé, celle de Diderot avec Sophie Volland par exemple, et des journaux, des mémoires sans artifice comme ceux de Samuel Pepys ou de Mme de Boigne. Personnellement, je comprends très bien l'intérêt du public pour ce genre d'écrits.

KS : En préface aux Lettres à Sartre, vous écrivez: "Quand, en 1983, Simone de Beauvoir publia les lettres de Sartre, ses amis s'étonnèrent 'Mais les vôtres, Castor?'. " À cette question, écrivez-vous encore, elle a opposé cette réponse: "[J] e ne trouve pas que, de mon vivant, je devrais publier des lettres de moi. Quand je serai morte, peut-être, si on les retrouve, on pourra les publier » (9). Or, en 1986 [quelques mois après la mort de Simone de Beauvoir], vous les avez retrouvées. "En fouillant sans but au fond d'un placard", dites-vous, vous avez découvert "un paquet massif des lettres et des lettres de sa main, la plupart encore pliées dans les enveloppes ". Pourriez-vous revenir plus longuement sur la scène de cette découverte, si ce n'est pas trop vous demander?

SLB : En 1986, j'ai été anéantie par sa disparition. Ça ne peut pas se raconter, à peine se dire. J'allais souvent rue Schœlcher chez « le Castor », cela m'était nécessaire, et en même temps, une fois arrivée là, je tournais en rond, je m'enfuyais. Mais je savais que je devais commencer à ranger, à trier cette masse de manuscrits, de papiers, de livres, d'objets, à ouvrir les placards. J'ai fini par m'y mettre, en me forçant, puis au bout de plusieurs mois, systématiquement. Un jour, en fouillant un placard au-dessus de son bureau, je suis tombée sur une boite, tout au fond, qui renfermait ses lettres à Sartre. Comme elle était dissimulée derrière un rempart d'autres dossiers, de manuscrits, cela peut expliquer que Simone de Beauvoir les ait crues égarées. II se peut aussi qu'elle n'ait pas eu vraiment envie de les retrouver lorsqu'ensemble nous faisions des rangements. J'ai été bouleversée, j'ai tout de suite compris l'importance de cette découverte. En ce qui me concerne, c'était le salut : j'allais travailler, j'avais un but immédiat et concret qui m'arrachait à la gratuité et qui de plus me permettait de la retrouver. Parce qu'on sent tellement sa présence dans ces pages spontanées.

Les lettres étaient restées tassées, pliées, souvent dans l'enveloppe originale. D'abord il a fallu les déplier, les dater, les classer. J'ai pris soin, évidemment, de garder les enveloppes qui sont en elles-mêmes des documents irremplaçables, avec les adresses et les divers cachets de la poste. Cette édition des Lettres à Sartre a été la première que j'ai faite, et j'y trouve beaucoup à redire. II faudrait la refaire en y intégrant de nombreuses lettres inédites, qu'au cours des années qui ont suivi j'ai découvertes, dispersées un peu partout dans les archives. Aussi bien de Simone de Beauvoir à Sartre que de lui à elle, et des plus 
Schwerdtner, Karin. « Les lettres-objets de Simone de Beauvoir : entretien avec Sylvie Le Bon de Beauvoir. » Nouvelle Revue Synergies Canada, №13 (2020)

signifiantes. Je songe à une lettre de Sartre, de 1948, où il s'explique, chose rare, sur le sentiment qu'il éprouve envers elle, sur la nature du lien unique qui les unit. C'est très émouvant.

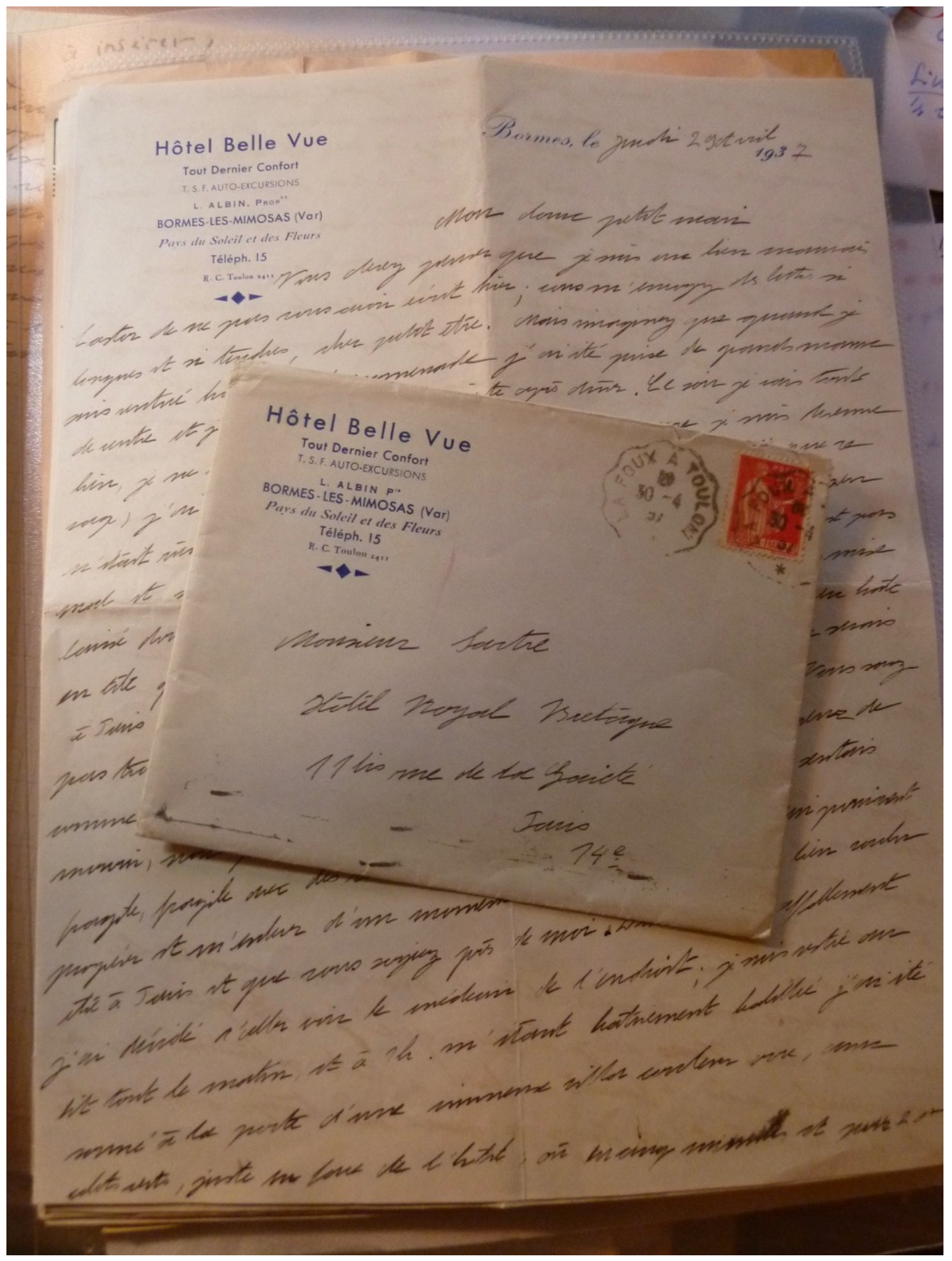

Lettre à Sartre 1937. Collection privée de Sylvie Le Bon de Beauvoir. Photo reproduite avec sa permission.

\section{KS : Combien de temps vous a-t-il fallu pour classer ces archives?}

SLB : Vingt ans. J'ai acheté l'étage où nous sommes - j'habitais déjà au-dessus - pour pouvoir y entreposer les archives. Au début je ne savais par où commencer. En gros il y avait des manuscrits, d'elle et de Sartre, énormément de correspondances de tous deux, il y avait les archives photographiques et un tas de documents variés. Tant que Simone de Beauvoir habitait à l'hôtel, elle entreposait certaines choses chez sa mère ou chez Sartre - beaucoup malheureusement ont été détruites, entre autres le manuscrit de 
L'invitée, dont sa mère a couvert des pots de confitures... et quantité d'autres pulvérisés lors de l'attentat commis pendant la guerre d'Algérie chez Sartre. À partir du moment où elle a loué, puis acheté un logis à elle, c'est-à-dire à peu près à partir de la parution du Deuxième sexe, elle a tout conservé, mais sans ordre. J'avais la gorge serrée face à ce trésor en désordre, j'étais submergée. Les photographies, en particulier, m'ont tourmentée : je ne comprends pas que la plupart des photographes ne les datent pas, c'est tellement indispensable! J'ai tout étalé par terre, et j'ai plongé, en répartissant dans des boites. Je continue aujourd'hui à raffiner, à recevoir des documents nouveaux, mais l'essentiel est fait, je m'y retrouve facilement, c'est même gratifiant. Bien sûr, classer pose en soi des problèmes, c'est très personnel, un classement, les catégories choisies peuvent s'entrecroiser, elles restent en partie arbitraires, mais quand une boite ne convient pas, je cherche dans une autre, je me fais confiance! Cela explique - ce que souvent les gens ne comprennent pas, ils s'étonnent - qu'on ne peut déléguer ce travail, ni se faire aider, sauf pour des tâches secondaires, parce que classer n'est pas un simple mécanisme objectif. II est certain que si ce travail d'archivage n'avait pas été achevé, l'édition des Mémoires dans la Bibliothèque de la Pléiade n'aurait pu se faire. Avant tout, je n'aurais pu me charger de la Chronologie, qui m'a pris quinze ans, car j'ai tout voulu vérifier de première main, en repartant de zéro, pour qu'elle fasse référence. On trouve des chronologies de Simone de Beauvoir un peu partout, dans des biographies, ou sur internet, toutes sont bourrées d'erreurs, chacune reproduisant celles des autres. Ensuite, l'équipe de la Pléiade devait pouvoir disposer des manuscrits, ce qui à soi seul représente un énorme volume où il fallait s'orienter (quatre ou cinq versions autographes de La Force des choses, par exemple). J'ai pu ainsi assurer l'établissement du texte - immense travail invisible. Enfin, il fallait pouvoir maîtriser l'ensemble des documents existants, y compris les photos, pour l'Album Pléiade, qui est une biographie illustrée. J'ai rédigé le texte et parallèlement, pendant plus d'un an, les iconographes Gallimard sont venus chez moi tout pré-photographier. Dans cette richesse foisonnante, un choix a été opéré, puis un professionnel est venu ici assurer la finalisation en très haute qualité. Les illustrations de l'album sont, je crois, riches et réussies dans leur variété, elles vont des portraits d'art signés de grands noms de la photographie aux clichés d'amateurs des albums de famille, de pages autographes des manuscrits aux couvertures des traductions dans le monde entier, du chèque du Prix Goncourt attribué au roman Les Mandarins en 1954 (si infime que la romancière ne l'a pas touché) à d'anciens passeports, à des emplois du temps de professeur, d'une statuette de Giacometti à des dessins de Sartre...

\section{KS : Entre les lettres à « donner » et celles à conserver chez vous, comment avez-vous fait le tri?}

SLB : Les circonstances ont joué un rôle. En 1986, en tant qu'héritière de Simone de Beauvoir, l'État m'a réclamé des droits de successions considérables, que j'étais incapable de payer. II faut savoir que le nom de Simone de Beauvoir est en quelque sorte coté par le fisc comme une valeur en Bourse. Pour m'acquitter en partie, j'ai proposé de déposer à la Bibliothèque nationale de France un certain nombre de manuscrits, tant de Simone de Beauvoir que de Sartre. On appelle cela une " dation ». J'ai offert alors, entre autres, la totalité de la correspondance Beauvoir-Sartre.

Plus tard, j'ai donné bénévolement l'ensemble du courrier des lecteurs de Simone de Beauvoir. Quoiqu'il ne soit pas dans le domaine public, chacun peut le consulter, je donne toujours l'autorisation. D'une manière générale, Simone de Beauvoir souhaitait que ses écrits et ceux de Sartre reviennent à la BNF et, dans la mesure où cela dépend de moi, ce sera le cas.

J'ai conservé les correspondances d'intimes, d'amis de jeunesse ou de toute sa vie, de célébrités. Dans cette dernière catégorie, deux charmantes lettres de Jean Cocteau envoyées après L'Invitée et les Mémoires d'une jeune fille rangée. Parmi les correspondances durables, je citerais celles avec des femmes ou des hommes, écrivains ou désireux d'écrire, comme Violette Leduc, Thérèse Plantier, Claire Cayron, Jean Donostia. Certaines appartiennent à des musées ou des bibliothèques, ainsi celle très intéressante échangée avec la romancière Thérèse Plantier appartient au Musée des Lettres et des Manuscrits, qui a été récemment l'objet d'un scandale financier et dont le fonds a été mis sous séquestre. Heureusement j'avais tout consulté juste avant. Et comme je vous l'ai dit, on ne cesse de m'en envoyer.

KS : Dans son texte consacré à " L'écriture de soi ", Foucault disait qu'écrire, c'est "'se montrer', se faire voir, faire apparaître son propre visage auprès de l'autre " (425). Pour votre part, vous suggérez que, dans ses Lettres à Sartre, "la personne réelle " (10) de Simone de Beauvoir surgit. De ce point de vue, peut-il y avoir quelque chose de risqué, pensez-vous, à publier intégralement ses lettres?

SLB : Simone de Beauvoir n'était pas femme à reculer devant le risque. Le fait d'écrire est déjà un très grand risque, comme en témoigne votre livre d'entretiens avec des écrivains, Le (beau) risque d'écrire ... Elle s'est « montrée » sans armure dans ses mémoires, elle s'est jetée toute vive dans ses romans. Oui, il y a quelque chose de risqué lorsqu'on publie des correspondances, ou des journaux, mais si on publie, il faut publier intégralement, à mon sens. Tout ou rien. Simone de Beauvoir et moi, nous aimions beaucoup 
la collection "Le temps retrouvé » [aux éditions Le Mercure de France] qui publie souvenirs, journaux, récits de voyage, mémoires et correspondances. Quelle frustration lorsqu'on avait pratiqué des coupures ! Cela nous paraissait une erreur et une faute. Bien entendu, cet avis peut ne pas être partagé par tout le monde. Et je crois que l'intérêt des correspondances n'est plus à démontrer, il ne cesse de croître, comme vous le dites. Ce sont des témoignages essentiels sur le vécu authentique d'individus, célèbres ou non, c'est la résurrection d'époques révolues. C'est la transmission d'un lien vivant nécessaire entre le passé et le présent. Présent qui ne cesse de s'anéantir dans le passé...

KS : Vous dites n'avoir pas de doutes sur l'intérêt de publier les correspondances de Simone de Beauvoir, même si, comme vous l'admettez vous-même, votre avis peut (ou a pu) ne pas être partagé. Au moment de leur parution, par exemple, les Lettres à Sartre ont « fait des remous » (Simonet-Tenant 99)...

SLB : Oui, c'était en 1990, peu de temps après la disparition de Simone de Beauvoir. Circulaient encore beaucoup d'idées reçues à son égard, de poncifs et de stéréotypes aberrants, par exemple qu'elle était quelqu'un de froid, de rigide, de sec. Préjugés dus en partie à son statut d'intellectuelle, qui pour beaucoup d'hommes et certaines femmes, est un terme péjoratif. Aussi, quand lecteurs et critiques l'ont « découverte » en chair et en os, ils se sont étonnés, voire même offusqués, comme si bousculer leurs a priori était inconvenant. Moi, je me suis étonnée de leur étonnement, de cette soi-disant " découverte », des « révélations " qui leur arrivaient, car il suffit de la lire, mais ce qui s'appelle vraiment lire, pas parcourir superficiellement, pour dans ses romans et ses mémoires tout découvrir de sa personne. Entre ses œuvres et sa correspondance, il n'y a pas de hiatus, il n'y a pas deux femmes en elle, comme on l'a dit sottement. II est vrai que Simone de Beauvoir a vécu, pensé et écrit avec hardiesse, en femme libre, hors des normes et des conventions, et qu'en 2019 elle est toujours loin devant nous.

Mais les choses ont bougé. Depuis 1990, l'atmosphère, l'accueil, la compréhension ont changé, et positivement. Je n'ai pas rencontré d'hostilité, au contraire, durant les trois mois de 2018 où j'ai contribué à la télévision, à la radio, dans les journaux, à la promotion des deux tomes de la Pléiade et de l'Album. On a pu parler de points intéressants, littérature, féminisme, voire philosophie, sans immédiatement retomber dans des contresens décourageants.

\section{KS : Pour terminer : avez-vous des projets? Une édition de lettres de jeunesse pourrait-elle vous tenter?}

SLB : Oui, et pas seulement de jeunesse - celles-là sont nombreuses, il est vrai, elle-même parle d'une " tempête épistolaire » durant l'été de ses 19 ans. II y a celles à son amie Zaza, à Olga Kosakiewicz, à Merleau-Ponty. J'ai mentionné les lettres à sa mère, à Violette Leduc, à d'autres, qui débordent largement sa jeunesse. Mais, avant tout, je veux m'occuper de la suite de la Pléiade, un tome de romans et un autre des essais théoriques, avec Le deuxième sexe, qui me parait l'analogue des deux autres grands classiques de la libération parus dans cette collection, La Critique de la raison pure, et Le Capital.

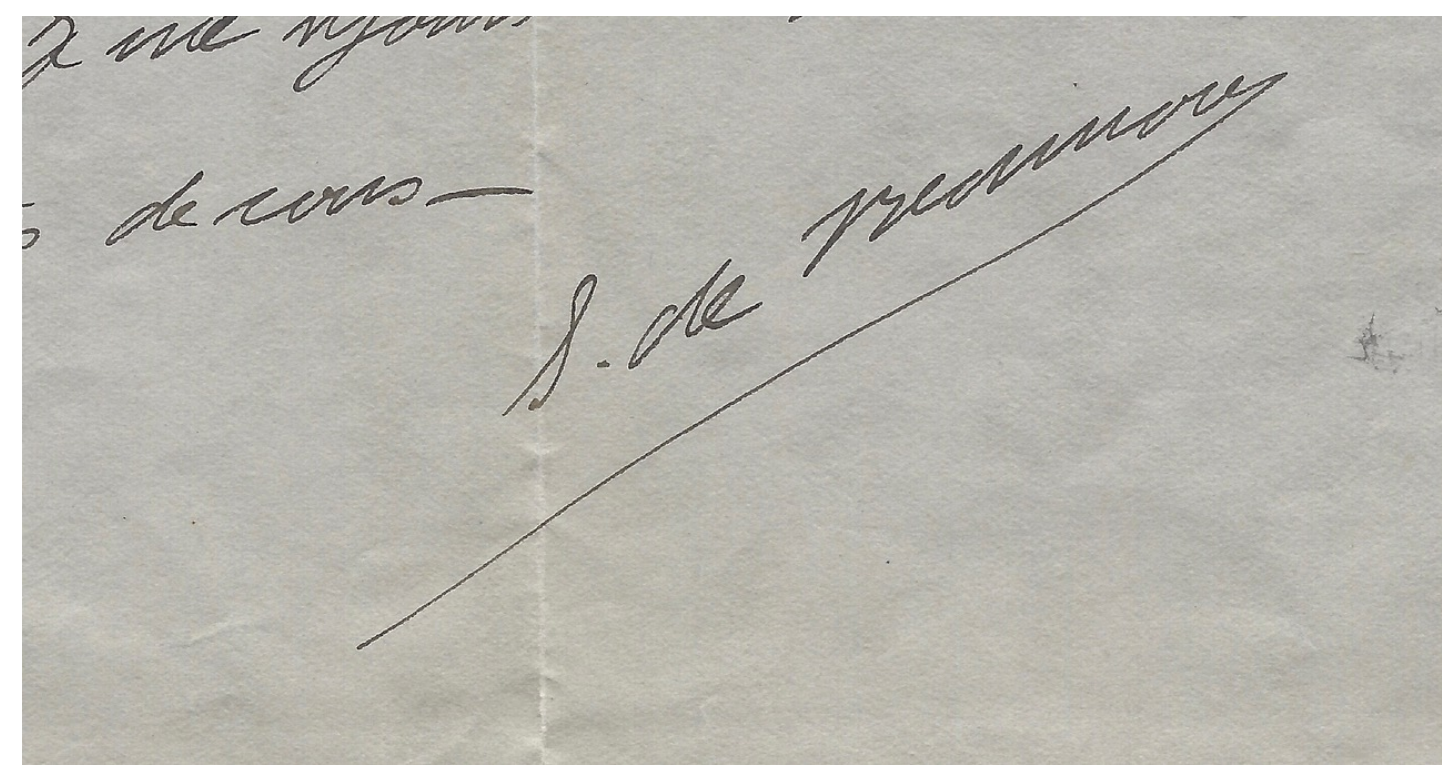

Signature de Simone de Beauvoir. Photo reproduite avec la permission de Sylvie Le Bon de Beauvoir. 


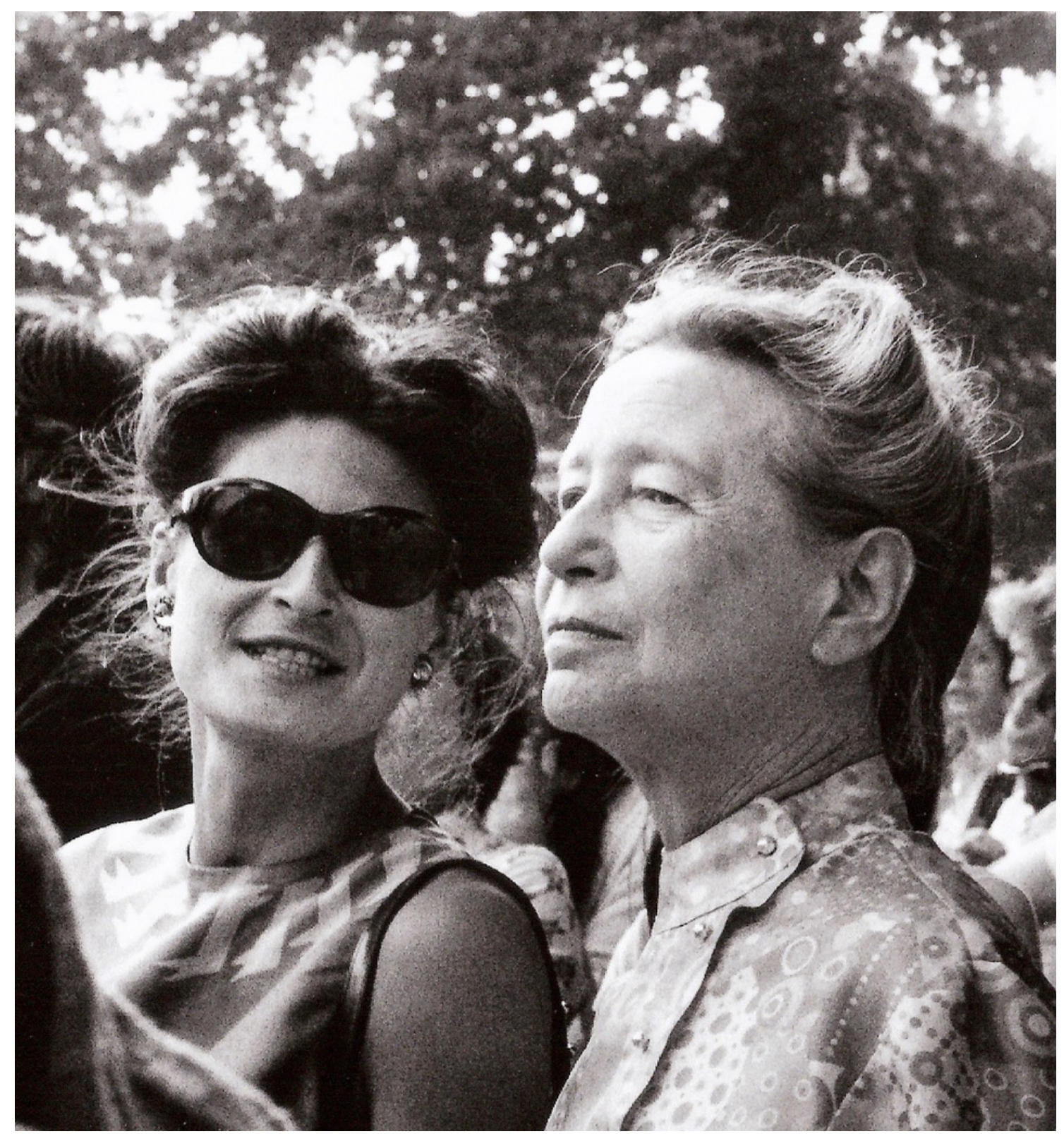

Sylvie Le Bon et Simone de Beauvoir, le 17 juin 1973. Collection privée de Sylvie Le Bon de Beauvoir.

\section{Notes}

${ }^{1}$ Nous remercions très sincèrement Sylvie Le Bon de Beauvoir de sa précieuse collaboration et d'avoir bien voulu nous proposer quelques photos pour publication. Nous remercions également Fanny Leveau, doctorante en études françaises à l'Université Western, pour sa transcription de propos recueillis.

2 « [O]n assiste à une véritable expansion des études épistolaires, situées dans de nombreux centres de recherche. Si la lettre est un genre très à la mode, c'est aussi que cette promotion de l'épistolaire tient à un double intérêt critique, textuel et culturel » (Jovicic 1).

${ }^{3}$ Sylvie de Beauvoir explique dans notre entretien, ainsi que dans celui que nous citons ici, que « [m]ettre de l'ordre dans toutes ces archives [lui] a pris des années. [Elle a] même dû agrandir [s]on appartement pour leur consacrer une pièce! » (Frey). 
${ }^{4}$ Au sujet des lettres reçues de lecteurs, voir les travaux de Marine Rouch. Voir aussi : Alice CaffarelCayron, "The influence of Simone de Beauvoir's Writings on Claire Cayron's Personal and Creative Life".

${ }^{5}$ Ce sont les propos de Sylvie Le Bon de Beauvoir, tels que recueillis par Pascale Frey.

${ }^{6}$ Nous citons à ce sujet la traductrice, critique littéraire et éditrice Claire Debru (Schwerdtner : 258).

\section{Ouvrages cités}

Beauvoir, Simone de. Lettres à Nelson Algren. Gallimard, 1997.

---. Lettres à Sartre, tomes I et II. Gallimard, 1990.

---. Mémoires, tomes I et II. Sous la direction de Jean-Louis Jeannelle et Eliane Lecarme-Tabone, Gallimard, « Bibliothèque de la Pléiade », 2018.

Beauvoir, Simone de, et Jacques-Laurent Bost. Correspondance croisée (1937-1940). Gallimard, 2004.

Bouchy, Florence. «Lettres à l'aimé(e), succès d'édition. » Le Monde des livres, 9 février 2018.

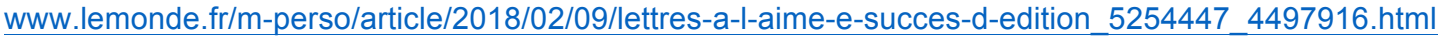

Caffarel-Cayron, Alice. "The influence of Simone de Beauvoir's Writings on Claire Cayron's Personal and Creative Life: A Preliminary Journey through their Correspondence (1964-1984)." Simone de Beauvoir Studies, vol. 29, 2013-2014, pp. 4-19.

Foucault, Michel. « L'écriture de soi. » Dits et écrits. 1954-1877, Gallimard, 1994.

Frey, Pascale. "Simone de Beauvoir reste très présente. 》 Tribune de Genève, 23 mai 2018. www.tdg.ch/culture/livres/Simone-de-Beauvoir-reste-tres-presente/story/22467808

Jovicic, Jelena. L'intime épistolaire (1850-1900) : genre et pratique culturelle. Cambridge Scholars, 2010.

Pomel, Fabienne (sous la direction de). Lire les objets médiévaux. Presses universitaires de Rennes, 2017.

Rouch, Marine. "Merci pour le message d'espoir": Ces femmes qui écrivaient à Simone de Beauvoir. » Le Magazine littéraire, avril 2016, p. 79.

Rouch, Marine. " "Vous êtes descendue d'un piédestal". Une appropriation collective des Mémoires de Simone de Beauvoir par les femmes (1958-1964). » Littérature, $\mathrm{n}^{\circ} 191$, sept. 2018, pp. 68-82.

---. « "Vous ne me connaissez pas mais ne jetez pas tout de suite ma lettre". Le courrier des lecteurs et lectrices de Simone de Beauvoir. " Genre de l'archive. Constitution et transmission des mémoires militantes, sous la direction de Françoise Blum, Codhos, 2017, pp. 93-108.

Schwerdtner, Karin. Le (beau) risque d'écrire. Entretiens littéraires. Nota bene, 2018.

---. «La lettre "en soi”. Entretien avec Claire Debru. » Risques et regrets, Les dangers de l'écriture épistolaire, sous la direction de Geneviève De Viveiros, Margot Irvine et Karin Schwerdtner, Nota bene, 2015, pp. 251-264.

Simonet-Tenant, Françoise. "Correspondances et journaux de femmes: une fonction mémorielle ? " Revista do Instituto de Estudos Brasileiros, $n^{\circ}$ 68, 2017, pp. 84-100. 